\title{
9 Christian perspectives on informed consent
}

\author{
Laura Palazzani
}

\section{Informed consent: general ethical principle from the Christian perspective}

The Christian perspective in bioethics, in the framework of a creationistic concept of nature with a normative value (finalism) and in a belief to know the truth in nature (cognitivism), considers human life as worthy of respect and protection (as a gift from God and created in His image) since it is the expression of personal life, dynamically intent on fully manifesting itself.

The principles proposed in bioethics are the following: the defence of human life's objective and absolute value, its intangibility and the impossibility to dispose of it arbitrarily; the therapeutic principle, according to which any intervention on life is justifiable only if it aims to cure the subject (to save lives and improve health); the principle of freedom and responsibility, where freedom denotes an objective limit to respect the lives of others; the principle of justice and solidarity or attaining common good through the good of the individual and solidarity towards those in need according to their particular vulnerabilities. ${ }^{1}$

Informed consent acquires a specific meaning in this conceptual framework. The Catholic Medical Association and National Catholic Bioethics Centre published a document entitled "Catholic Principles and Guidelines for Clinical Research,"2 which made references to the concept of informed consent in both clinical practice and research. The basic understanding of Christianity regarding informed consent is

1 The physician's duty to inform and tell the truth in a complete, clear and comprehensible way about the health of the patient and the possibility to be cured (prevention, diagnosis and therapy) and cared for. The physician should be neither directive nor descriptive/neutral towards the patients but help them make conscious decisions oriented towards proportionate protection and respect for life. There is no place for 
intentional falsehoods, misleading statements or bias. It is the responsibility of everyone, especially a physician or a medical researcher, to respect the sacred and inviolable right to life of the human subject at every stage from its first formation to death. In this regard, the obligations of the natural moral law and Catholic moral teaching must always be respected. The medical researcher and those who assist in medical research must strive to do good (principle of beneficence) and avoid causing deliberate harm to the subjects in any possible way (principle of non-maleficence).

2 The duty of the patient to be informed to be aware of the decision. When there is a trusting relationship with the physician (a sort of "conscious paternalism" requested by the patient), there may also be a right not to be informed in specific circumstances. In both cases, the Christian perspective emphasizes the duty to accept the cure and care of the physician. In this sense, the patient's conscious, free and autonomous choice is respectful of life's value when it is orientated towards the objective good: the conservation of life and health improvement by accepting appropriate treatment. In the case of minors and those who lack the capacity to consent (incapacitated), either parents or surrogates may offer decision-making according to their best objective interests. The priority of the objective value of life is derived from the natural law precepts to conserve life. In this context, there may be a difference between the Protestant (more open to subjectivity) and the Catholic perspectives (more linked to the objectivity of the ethical choice). ${ }^{3}$

3 Informed consent for both the physician or health professional and the patient should (in a framework of human dignity and personal integrity) respect the principles of beneficence (priority of the good for the patient) and non-maleficence (not to harm the patient). These principles refer to the criterion of risk-benefit proportionality: the potential (direct or indirect) benefits of any study must be weighed against potential risks (concerning integrity, health and well-being). When a particular intervention does not directly benefit a minor or members of a vulnerable population, the associated risks must be relatively insignificant (a burden, reduced to a minimum). Research against the good of the person is immoral. High risk is unacceptable. Adequately verifying all the benefits and risks involved in the intervention is essential.

4 In this respect, the principle of responsibility should guide the choice of both the patient and the physician. The physician has therapeutic responsibility towards the patient, and the patient or subject has personal responsibility towards one's life and health and social responsibility for the good of science and society. Regardless of physical-psychic or social condition or medical need, everyone must be fully and adequately 
protected as a person worthy of respect. Generally, both the human subject and the researcher must recognize the moral obligations under natural law deriving from the value of life and be responsible for it. ${ }^{4}$

5 The principle of autonomy. The physician must respect the spirituality and religious beliefs of the human subject. The physician should not compromise these beliefs without failing in some moral duty of the natural law. If the patient refuses an appropriate treatment (or asks for inappropriate treatments), the physician should try to convince him to be cured but cannot impose his view. Conversely, in case of a confident refusal, grounded in religious or rational/emotional motivations, he needs to accept it. Verbal coercion or forceful persuasion is not acceptable. However, based on the assumption that patients are, sometimes, in too much pain and are not objective enough to make the right moral decision, medical staff and family members can withhold certain information and persuade the patient to make the best moral choice. Free and informed consent should not be individualistic as if the patient were an isolated decision-maker. Instead, it should embed the patient in a relational context that supports and properly orients his choices. He or his surrogate should have access to medical and moral information and counselling to form her conscience. Free and informed healthcare decisions should be followed if they do not contradict Catholic principles. If a request for treatment goes against the moral conscience of the physician, he could object (conscientious objection). The medical researcher has the moral responsibility to act with a properly formed conscience and withdraw from a scientific intervention or investigation rather than act against his judgement of conscience.

6 Informed consent is inspired by Jesus, who cured the sick with compassion, generosity, and understanding. Christians believe that disease and suffering are trials from God to bring them closer to salvation through the death and into His grace. Scientific research should be done to serve those who are ill, not solely or primarily for the benefit of the researchers. Research should be conducted according to accepted scientific principles, and it should be deemed necessary and potentially useful for the patient. We cannot subject an individual to unnecessary or disproportionate risks which exceed the research's expected benefit. The researcher must never participate in projects that may involve treating the human subject as an object of interest. Studies that may involve immoral cooperation with evil must be avoided. The subjects may choose to accept risks or sacrifice themselves (especially in experimentations where risks are higher) for the good of others and society out of solidarity or charity. However, we need to protect the subject's 
integrity by verifying authentic intention and the absence of any coercion or pressure.

In this perspective,

The Church respects and supports scientific research when it has a genuinely humanist orientation, avoiding any form of instrumentalization or destruction of the human being and keeping itself free from the slavery of political and economic interests. In presenting the moral orientations dictated by natural reason, the Church is convinced that she offers a precious service to scientific research, doing her utmost for the true good of the human person. In this perspective, she recalls that, not only the aims, but also the methods and means of research must always respect the dignity of every human being, at every stage of his development and in every phase of experimentation. ${ }^{5}$

\section{Informed consent and conditions of vulnerability}

The principle of precaution/prudence is generally applied in the Christian perspective. Above all, it must be applied in case of conditions of vulnerability because of the severity of the illness, age (minors, elderly), sex (men/ women), socio-cultural conditions (indigent, immigrants). ${ }^{6}$

\section{Informed consent of the incapable person and minors}

In the case of minors who are legally not capable of understanding and making decisions, their involvement is justified by a proportionality between the foreseeable risks and benefits in the framework of good and relevant research. Experimentation that is not expected to provide direct benefits for the minor, but only for other persons in similar conditions (in terms of age, type of illness and other characteristics), can be ethically justified when it is not possible to obtain the same results through experiments on adult, competent subjects and the risks and burdens are minimal. ${ }^{7}$

On the basis of the principle of equality and justice, just like any other human being, children have the right to receive drugs that will guarantee health in the same way adults do. It would not be ethical to exclude children from trials since it would mean discriminating against their interests and fundamental rights to life and health.

One of the most critical elements of clinical trials in children is informed consent. The need to obtain the consent of both parents is an established bioethical practice. Concerning the general information before consent, the investigator must evaluate the parents' real motives to accept their child's 
recruitment in a trial and exclude ethically unacceptable reasons. For example, to benefit from medical treatment that is otherwise not guaranteed or obtain greater attention by the doctors in the treatment of the children.

The parents' consent should be accompanied by the child's assent, which is proof of their actual involvement in the medical decisions, together with their parents. The appropriateness of the information will be evaluated case by case according to cultural and social context and the existential context since each child has a different evolution and maturity and can react differently to illness or pain.

The minor should receive information from expert personnel proportional to their capacity to understand the risks and benefits according to age and maturity. Furthermore, the investigator should consider the desire expressed by the minor to take part in the experimentation or withdraw from it at any moment. The child should be told that their desires are important in the decision, making it clear that they cannot be decisive alone. Specific attention should be paid so that the child's involvement is not an indirect insistence on participation, which should always be free and unconditioned by external factors. The conditioning is particularly problematic in a paediatric phase given the child's vulnerability from the external influences of adults, members of the family and doctors. In the context of assent, doctors should help the child to understand the aim of the trial, the procedures forthcoming and the experiences that they will have. They should try to perceive how much the child has understood and what are their often-unexpressed concerns are to help participants to overcome them. The best interests of the children is the principal Christian value in clinical practice and research.

A challenging element is the involvement of healthy or sick children as a control group or as subjects of "non-therapeutic" experimentation. They will not receive any direct benefits but only indirect ones by helping other children with identical pathology in the future. Non-therapeutic experimentation on minors cannot be excluded if significant improvements in scientific knowledge were to be achieved with a positive willingness and minimum risk or discomfort.

Disputes exist among Catholic moral theologians and bioethicists about whether proxy consent can ever be morally valid in non-therapeutic settings and, if it can, under what conditions and why. One of the most debated issues regards the concepts of minimal risk and discomfort in non-therapeutic research. Is it licit to subject the incompetent persons to non-therapeutic procedures involving minimal risk or a risk slightly exceeding minimal? If so, what benchmarks should be employed? If not, would any amount of risk in non-therapeutic experimentation render it morally objectionable? What degree of risk can be justified in non-therapeutic settings, and how is the risk-benefit ratio determined in each case? There is a need for a careful 
justification of the scientific relevance of the study and a detailed reflection on informed consent in Catholic anthropological and moral premises, which give priority to the dignity of the human person. ${ }^{8}$

In this context, it is indispensable that informed assent/consent exists regarding the risk and the entity of the risk. Christian perspective considers it legitimate to perform a non-therapeutic trial on a child with an actual capacity of informed assent and supported by the consent of the parents or legal representatives if there are no significant risks either for the life or for the physical integrity of the minor. The requisite of the minimum risk and or discomfort is ethically central.

It is a different bioethical evaluation to use experimental drugs as a last resort to save a minor's life with incurable terminal conditions (maybe also in a condition of imminent death) and in the absence of effective therapeutic alternatives. In such cases, the ethical decision should be proportionate to the actual circumstances to seek the conditions that are respectful of the dignity of the minor. In the first place, it is essential to gather objective scientific data about the seriousness of the illness (verification of the condition of impossibility of cure). Second, we need to consider the present and foreseeable future suffering, the patient's quality of life and the reduction of suffering.

Experimental pharmacological interventions which are aggressive and intensive are ethically licit and proper in the Christian perspective when there is a minimum "therapeutic hope" and when the likely suffering is proportionate to the potential benefits to improve the quality of life (or at least minimization of suffering), with the consent of the parents and possibly the assent of the minors. The suspension of aggressive and intensive experimental therapies is ethically licit and, sometimes, dutiful when life expectancy is short, the prognosis is undoubtedly poor and the therapies futile and harmful. In these cases of so-called therapeutic obstinacy, only ordinary treatment is given along with palliative care and human caregiving. Human and Christian solidarity justifies these gestures and gives them meaning and value.

\section{Research on women: informed consent of fertile, pregnant and breastfeeding women}

There are different reasons for underrepresentation and numerical inferiority of participation of women in clinical trials. Some reasons generally concern the way of considering experimentation and medicine. In experimentation, there is a tendency towards "generalization," a tendency towards "neutrality" and assimilation of women to men. These orientations conflict with the need for individual specification and gender differentiation, above all today, in the time of the so-called precision medicine. ${ }^{9}$ 
The possible pregnancy in childbearing age has led the pharmaceutical companies to exclude women from clinical protocols or impose specific hormonal contraceptives as a condition for participation in research because of the possible risks to the foetus.

From the Christian perspective, if clinical trials endanger the foetus's life or health recognized as a subject of rights, it is ethically preferable for women not to participate when such risks exceed the potential benefits to the women. Suppose the woman decides to enrol in the trial for social or personal aims. In that case, she should, nevertheless, be able to choose the modalities freely and responsibly to avoid pregnancy following her values and religious beliefs, among which abstinence from sexual relations, insofar as she should deem the use of contraceptives illicit owing to the scission between unitive act and procreation (as in the Catholic perspective). Using contraception also raises the problem of contra-gestation, which may impede not only the fecundation of gamete but also the process of implantation.

This particularly thorny issue requires a bioethical analysis that balances the needs of the trial with the values of the subjects taking part in the experimentation. The Christian bioethics perspective of informed consent will consider the gender difference and the moral principles of those taking part in the trials, offering women the possibility of sexual abstinence. If it is incompatible with the trial protocols, women will receive appropriate consultation to choose responsibly according to their moral and religious values. Informed consent should also be undersigned by her partner and include a variable time frame that can be extended even after the trial. ${ }^{10}$

Another specific bioethical issue in clinical research involves pregnant women. In this context, physicians often prescribe drugs for pregnant and breastfeeding women without studies or evidence of safety and efficacy under those conditions. Such treatments may include medications that can cause serious harm for both the woman and the foetus. The exclusion of pregnant women from clinical trials is the cause of the lack of data on potential benefits and harms to women and their future children. Therefore, we need to design research protocols for pregnant and breastfeeding women to determine potential risks and benefits.

Clinical research on pregnant women needs specific ethical requirements. Research with a potential direct benefit is only allowed when it cannot be carried out on non-pregnant and non-breastfeeding women. Risk-benefit assessment must consider the specific pregnancy situation, extend it to foetuses and even include the preconception staged. In such research, the criteria of minimal risk and minimum burden are compulsory for both the woman and the child. "Minimal risk" refers to the degree of harm or discomfort which should not be greater than those experienced in daily life or 
routine physical or psychological examinations. Research ethics committees require specific attention and prudence. In any event, evidence from prior animal experimentation is necessary. ${ }^{11}$

According to the Christian perspective, pregnant or breastfeeding women should not participate in non-therapeutic research that carries more than minimal risk to them and the foetus or infant unless the experiment is intended to explain pregnancy or lactation problems when there are no alternatives. When the research during pregnancy carries more than minimal risk, the woman should participate in follow-up evaluations to assess the effects on her and her foetus or child. She should be informed of the risks that participation may have on her health and the embryo, foetus and infant. If new scientific information arises during the research, this information should be immediately given to participants. At any stage of the research, the subject's right to withdraw consent should be respected. Follow-up of the pregnancy, the foetus and the child is essential, even months after the study.

\section{Research in a multicultural setting: intercultural informed consent}

Special attention must be given to vulnerable persons in clinical experimentation because of dependence (students, prisoners, military service personnel), social insecurity or poverty (the homeless, the unemployed, immigrants) and lack of education. These vulnerable situations could make it difficult to obtain valid informed consent.

Research carried out in emerging or developing countries should have clinical and scientific objectives that directly and specifically concern the local population. The scientific and ethical criteria used to evaluate and conduct these experiments must be the same as those conducted in developed countries. This research must respect local traditions and cultures and be approved in advance by either a national ethics committee of the sponsoring country or the local ethics committee. Researchers may carry out clinical experiments, especially those dealing with severe pathologies with no proven treatment. Experimental treatments could also involve women and men of childbearing potential, with possible risks when pregnancy occurs. ${ }^{12}$

Applying general ethical standards of clinical trials to the different cultural contexts, particularly in developing countries, needs interpretation and specification. The Christian priority towards human dignity and justice necessitates additional safeguards to avoid exploitation or abuse of vulnerable populations due to poverty, lack of education and understanding of scientific issues, lack of technical skills, scarce resources, disease and inability to have access to essential health products and services.

Community consultations might help the process of interpretation to acquire a better knowledge of local culture and involve community 
representatives in elaborating research projects. In this context, the role of the cultural mediator is essential. The aim is neither to impose foreign ethical standards (in a sort of ethnocentric imperialism or paternalism) nor to adapt to local standards (in a pragmatic relativistic attitude). It is meant to apply generally recognized Christian principles and values and seriously consider the specific culture's conditions and needs with an intercultural and interreligious approach.

Explicitly or implicitly, extra ethically required standards include the following: direct relevance of the clinical trial, equity in enrolment, tailored informed consent, proportionality and compensation for risks and damages and training and assistance to develop "collaborative partnerships."

Informed consent should be tailored to local customs, verifying that it is voluntary and freely given without coercion, incentives or "undue inducements." It may be oral and witnessed for the illiterate, with community leader's permission or family involvement in specific circumstances. In developing countries, participation in a trial could be an incentive to obtain food and primary healthcare and affect their voluntariness and presence of "undue" influence. The socio-economic conditions could push these countries to participate in the research without adequate awareness of the risks.

Another problem consists of some populations' difficulty to grasp the concept of research, which tends to be confused with care and assistance (the so-called therapeutic misconception). Involvement of other persons in formulating informed consent is acceptable only if we can verify the genuine awareness of individual participation (as well as the possibility to withdraw from it) and an absence of direct or indirect external pressure. This awareness should be verified as being personal and cannot be substituted by someone else.

Confidentiality is another issue connected to informed consent. It may be weakened (or obliterated) with the family's possible involvement in the process of granting permission to carry out the research. Some cultures do not have a concept of "privacy." It raises an ethical problem because participation in research may, for some vulnerable populations, carry the stigma of being sick. In such contexts, cultural associations may play a supportive role to help the patients not to be marginalized.

In the Christian perspective, the principles of justice and solidarity require participants to receive appropriate treatments that yield potential benefits. They should, otherwise, be compensated for any harm directly related to participation in research. As an expression of international cooperation and solidarity, healthcare infrastructures are needed to support proper distribution. They guarantee continued access to post-trial benefits and treatment to participants and populations outside the research context of the country where the trial is conducted. It means that protection should be 
provided through arrangements of mandatory insurance because of possible damages, where the premium is assessed according to the local economic situation. This arrangement could be guaranteed by non-profit and internationally accredited independent organizations, which may monitor this ethical requirement.

An ethical requirement from the Christian perspective is to help developing countries build the capacity to become fuller partners in international research on both scientific and ethical levels, enhancing collaboration and creating an atmosphere of trust and respect. There should be a guarantee of assistance to developing countries during the experimentation without inflicting on them the burden of the "indirect costs" on an already precarious local health system. This collaboration will help them become full partners in international research and stimulate the improvement of the local health system by transferring technical and scientific skills. Involving doctors and representatives of the host country will help monitor compliance with ethical standards and avoid abuse. ${ }^{13}$

\section{Notes}

1 Kevin D. O'Rourke and Philip Boyle, Medical Ethics: Sources of Catholic Teachings (Washington, DC: Georgetown University Press, 1999); David F. Kelly, Gerard Magill, and Henk ten Have, Contemporary Catholic Health Care Ethics (Washington, DC: Georgetown University Press, 2013); Gilbert Meilaender, Bioethics: A Primer for Christians, revised ed. (Grand Rapids, MI: W. B. Eerdmans Publishing Co., 2004); Jason T. Eberl (ed.), Contemporary Controversies in Catholic Bioethics (Cham, Switzerland: Springer International Publishing AG, 2017); Anthony Fisher, Catholic Bioethics for a New Millennium (Cambridge: Cambridge University Press, 2011); Padraig Corkery, Bioethics and the Catholic Moral Tradition (Sedona, Arizona: Veritas Publishing, 2011); The National Catholic Bioethics Centre, A Catholic Guide to Ethical Clinical Research (Philadelphia, PA: The Catholic Medical Association and the National Catholic Bioethics Centre, 2009); James J. Walter and Thomas A. Shannon, Contemporary Issues in Bioethics: A Catholic Perspective (Lanham, MD: Rowman and Littlefield Publishers, 2005).

2 The Catholic Medical Association and National Catholic Bioethics Centre, "Catholic Principles and Guidelines for Clinical Research," National Catholic Bioethics Quarterly 7, no. 1 (Spring 2007): 153-165. For the Catholic perspective: Pontifical Council for Pastoral Assistance to Health Care Workers, Charter for Health Care Workers (Vatican City: Pontifical Council for Pastoral Assistance to Health Care Workers, 2016), 82-84. See United States Conference of Catholic Bishops, Ethical and Religious Directives for Catholic Health Care Services (Washington, DC: United States Conference of Catholic Bishops).

3 John Paul II, "To the Members of the Pontifical Academy for Life," Address (February 23, 2003): "The researcher must respect the dignity and nature of the human subject as having the powers of intellect and free will. This fact is the foundation for moral obligations regarding free and informed consent. Generally, both the subject and the researcher must recognize that the subject has moral 
obligations under the natural law concerning his or her life. The researcher must always be mindful that subjects have a natural law obligation to care for and conserve their health in a manner proportionate to the specific circumstances. At no time should a researcher attempt to compromise this obligation."

4 "All persons served by Catholic health care have the right and duty to protect and preserve their bodily and functional integrity. The functional integrity of the person may be sacrificed to maintain the health or life of the person when no other morally permissible means is available," see United States Conference of Catholic Bishops, Ethical and Religious Directives for Catholic Health Care Services, 29.

5 John Paul II, "To the Participants in a Conference Promoted by the Pontifical Commission for Pastoral Assistance to Health Care Workers," Address (November 12,1987). The Catholic theological and philosophical reflection on the topics in bioethics: Edmund Pellegrino, Daniel C. Thomasma, The Virtues in Medical Practice (Oxford: Oxford University Press, 1993); Elio Sgreccia, Personalist Bioethics: Foundations and Applications (Philadelphia: National Catholic Bioethics Center, 2012).

6 Laura Palazzani (ed.), "Special Issue on iConsent:-Improving the Guidelines for Informed Consent, Including Vulnerable Populations, under a Gender Perspective," Rivista di BioDiritto, Special Issue 1 (2019): 154.

7 Mazur Gregorz, Informed Consent, Proxy Consent, and Catholic Bioethics: For the Good of the Subject (Dordrecht: Springer Nature, 2012).

8 Ibid.

9 Italian Committee for Bioethics, "Pharmacological Trials on Women," Opinion (2008).

$10 \mathrm{Ibid}$., in the personal remarks at the end of the Opinion: "It is ethically acceptable or justifiable from a medical point of view, to force the inclusion of potentially fertile patients in pharmacological clinical trials to use contraceptive methods chosen and imposed by the sponsor and supported by binding clauses from insurance companies, in order to give the necessary economical guarantees to cover possible damages. This request, independently from ethical and religious positions, is not in fact in keeping with the responsible freedom of choice that - within completely personal choices like those regarding married life and the responsibility to procreate - the patient applying to enlist in clinical trials must take on in a personal and independent way, after an exhaustive conversation with the doctor experimenter. In addition, we highlight that contractual imposition from the sponsor of the trials to use contraceptive methods, gives ethical committees, which operate in health institutions regulated by particular rules, some evident difficulties in evaluating and accepting the protocols."

11 Laura Palazzani, "Review of Clinical Research Involving Pregnant Women by Françoise Baylis and Angela Ballantyne (eds)," Theoretical Medicine and Bioethics 40 (2019): 343-345.

12 John Paul II, "To the Members of the Pontifical Academy of Sciences," Address (October 23, 1982).

13 Italian Committee for Bioethics, "Pharmacological Trials in Developing Countries," Opinion (2011). 